\title{
Eddy-Current-Effect Homogenization of Windings in Harmonic-Balance Finite-Element Models Coupled to Nonlinear Circuits
}

\author{
Ruth V. Sabariego ${ }^{1}$, Korawich Niyomsatian ${ }^{1}$, and Johan Gyselinck $^{2}$ \\ ${ }^{1}$ KU Leuven, Dept. Electrical Engineering, Campus EnergyVille, Belgium \\ ${ }^{2}$ Université Libre de Bruxelles (ULB), BEAMS Dept., Belgium
}

\begin{abstract}
This paper deals with the harmonic-balance finite-element analysis of a multi-turn winding device coupled to electrical circuits comprising nonlinear components. The eddy-current effects in the windings are accounted for via a frequency-dependent reluctivity and impedance determined with a homogenization technique. The proposed multi-frequency approach is validated through a singlephase four-diode rectifier with an axisymmetric FE model of an inductor. The system of nonlinear algebraic equations is solved by means of the Newton-Raphson method. The harmonic-balance results are validated with classical time-stepping simulations. The computational cost is briefly discussed.
\end{abstract}

Index Terms-Eddy currents, finite element methods, harmonic balance, proximity effect, skin effect, nonlinear control systems.

\section{INTRODUCTION}

A CCOUNTING for the possibly non-negligible eddycurrent effects in the windings of electromagnetic devices requires a brute-force finite-element (FE) model with a fine discretization of each turn, which is too computationally expensive.

Frequency-domain homogenization techniques provide a closed-form continuous representation of the homogenized winding [1]-[3]. In [4], frequency-dependent proximity and skin effect parameters are identified in a general approach and straightforwardly included in a FE model by adopting: 1) a complex reluctivity in the homogenized winding; 2) a complex impedance in the electrical circuit. The method works with litz-wire bundles as well [5].

This flexible winding homogenization technique is further embedded in a harmonic-balance (HB) FE approach in [6].

In this paper, we aim at studying a homogenized multiturn winding coupled to an electrical circuit with nonlinear components via a HB-FE technique, which may be an efficient alternative to plain time-stepping in case of long transients [7]. Among the available implementations, e.g. [8]-[10], we adopt the Galerkin time-domain variant in [11]. The homogenized field-nonlinear circuit coupled model is validated by means of a single-phase four-diode rectifier and an inductor (Fig. 1).

\section{Generic (nONLINeAR) Field-Circuit ODEs}

Let us consider an electrical circuit that comprises a number of stranded and massive conductors (subscripts $S$ and $M$ ) in the FE domain (with e.g. a magnetic vector potential formulation) and a number of lumped resistances, inductances and voltage sources. The first-order ordinary differential equations (ODEs) arising from its FE discretization (with $n_{\mathrm{w}}$ weighing

Manuscript received June 27, 2017. Corresponding author: R. V. Sabariego (email: ruth.sabariego@kuleuven.be).

Color versions of one or more of the figures in this paper are available online at http://ieeexplore.ieee.org.

Digital Object Identifier xx.xxxx/TMAG.xxxx.xxxxxx. and test functions) in terms of the unknowns column vector $\mathbf{A}=\left[a_{1}(t) \cdots a_{n_{\mathrm{w}}}(t)\right]^{\top}$ features the reluctivity-dependent stiffness matrix $\mathbf{S}(\nu)$, the conductivity-dependent eddy-current matrix $\mathbf{T}_{M}(\sigma)$, and right handside column vectors accounting for the sources, i.e. $\mathbf{I}_{S}(t)$ with $n_{\mathrm{S}}$ currents in the stranded conductors (uniform current density) and $\mathbf{V}_{M}(t)$ with $n_{\mathrm{M}}$ voltages across the massive conductors (non-uniform current density due to skin and proximity effects):

$$
\begin{aligned}
& \mathbf{S} \mathbf{A}+\mathbf{T}_{M} \frac{\mathrm{d} \mathbf{A}}{\mathrm{d} t}=\mathbf{K}_{M}^{\top} \mathbf{R}_{M}^{-1} \mathbf{V}_{M}+\mathbf{K}_{S}^{\top} \mathbf{I}_{S}, \\
& \mathbf{V}_{M}=\mathbf{R}_{M} \mathbf{I}_{M}+\mathbf{K}_{M} \frac{\mathrm{d} \mathbf{A}}{\mathrm{d} t}, \\
& \mathbf{V}_{S}=\mathbf{R}_{S} \mathbf{I}_{S}+\mathbf{K}_{S} \frac{\mathrm{d} \mathbf{A}}{\mathrm{d} t},
\end{aligned}
$$

where $\mathbf{I}_{M}(t), \mathbf{V}_{S}(t)$ comprise $n_{\mathrm{M}}$ or $n_{\mathrm{S}}$ currents and voltages; diagonal matrices $\mathbf{R}_{M}$ and $\mathbf{R}_{S}$ comprise the respective resistances (dc value for the massive conductors); $\mathbf{K}_{M}\left(n_{\mathrm{M}} \times n_{\mathrm{w}}\right)$ and $\mathbf{K}_{S}\left(n_{\mathrm{S}} \times n_{\mathrm{w}}\right)$ are connectivity matrices.

By introducing $n_{l}$ independent loops and linked loopcurrent column vector $\mathbf{I}_{l}(t)$, the electrical circuit coupling is given by

$$
\mathbf{D}_{S l}^{\top} \mathbf{V}_{S}+\mathbf{D}_{M l}^{\top} \mathbf{V}_{M}+\mathbf{R}_{l} \mathbf{I}_{l}+\mathbf{L}_{l} \frac{\mathrm{d} \mathbf{I}_{l}}{\mathrm{~d} t}=\mathbf{V}_{l}
$$

where $\mathbf{D}_{S l}\left(n_{\mathrm{S}} \times n_{l}\right)$ and $\mathbf{D}_{M l}\left(n_{\mathrm{M}} \times n_{l}\right)$ are connectivity matrices (with 0, 1 and -1 elements, with $\mathbf{I}_{S}=\mathbf{D}_{S l} \mathbf{I}_{l}$ and $\mathbf{I}_{M}=\mathbf{D}_{M l} \mathbf{I}_{l}$ ), and with the inclusion of a number of lumped resistances, inductances and voltage sources via the square loop matrices $\mathbf{R}_{l}$ and $\mathbf{L}_{l}$, and the righthand side term $\mathbf{V}_{l}(t)$. Current sources and capacitances can also be considered. Alternatively, the modified nodal analysis can be adopted [12], [13].

Equations (1)-(4) can be gathered in the following system:

$$
\begin{array}{r}
\mathbf{M}(\mathbf{X}(t)) \mathbf{X}(t)+\mathbf{N}(\mathbf{X}(t)) \frac{\mathrm{d} \mathbf{X}}{\mathrm{d} t}=\mathbf{F}(t), \\
\mathbf{X}(t)=\left[\begin{array}{lll}
\mathbf{A} & \mathbf{V}_{M} & \mathbf{I}_{l}
\end{array}\right]^{\top}, \quad \mathbf{F}(t)=\left[\begin{array}{lll}
\mathbf{0} & \mathbf{0} & \mathbf{V}_{l}
\end{array}\right]^{\top},
\end{array}
$$


$\mathbf{M}=\left[\begin{array}{ccc}\mathbf{S} & -\mathbf{K}_{M}^{\top} \mathbf{R}_{M}^{-1} & -\mathbf{K}_{S}^{\top} \mathbf{D}_{S l} \\ \mathbf{0} & \mathbb{1} & -\mathbf{R}_{M} \mathbf{D}_{M l} \\ \mathbf{0} & \mathbf{D}_{M l}^{\top} & \mathbf{R}_{l}+\mathbf{R}_{S l}\end{array}\right], \mathbf{N}=\left[\begin{array}{ccc}\mathbf{T}_{M} & \mathbf{0} & \mathbf{0} \\ -\mathbf{K}_{M} & \mathbf{0} & \mathbf{0} \\ \mathbf{D}_{S l}^{\top} \mathbf{K}_{S} & \mathbf{0} & \mathbf{L}_{l}\end{array}\right]$,

with $\mathbf{R}_{S l}=\mathbf{D}_{S l}^{\top} \mathbf{R}_{S} \mathbf{D}_{S l}\left(n_{l} \times n_{l}\right)$ and $\mathbb{1}$ the identity matrix.

The nonlinearity in $\mathbf{M}$ may be due to a field-dependent reluctivity (e.g. reversible material law, $\mathbf{S}(\nu)$ ) or to currentdependent resistances (e.g. the on and off resistances of a diode, $\mathbf{R}_{l}\left(\mathbf{I}_{l}\right)$ ). A dynamic current-dependent lumped component can be included in matrix $\mathbf{N}$ (e.g a self, $\mathbf{L}_{l}\left(\mathbf{I}_{l}\right)$ ). The lumped resistances in $\mathbf{R}_{l}$ and inductances $\mathbf{L}_{l}$ are related to localized elements in diagonal matrices $\mathbf{R}\left(n_{\mathrm{R}} \times n_{\mathrm{R}}\right)$ and $\mathbf{L}\left(n_{\mathrm{L}} \times n_{\mathrm{L}}\right)$ by topological matrices $\mathbf{D}_{R l}\left(n_{\mathrm{R}} \times n_{l}\right)$ and $\mathbf{D}_{L l}\left(n_{\mathrm{L}} \times n_{l}\right)$ (1 if element in current loop, 0 otherwise), i.e. $\mathbf{R}_{l}\left(\mathbf{I}_{l}\right)=\mathbf{D}_{R l}^{\top} \mathbf{R}\left(\mathbf{I}_{l}\right) \mathbf{D}_{R l}$ and $\mathbf{L}_{l}\left(\mathbf{I}_{l}\right)=\mathbf{D}_{L l}^{\top} \mathbf{L}\left(\mathbf{I}_{l}\right) \mathbf{D}_{L l}$. Furthermore, the voltage in the $q$ th current loop due to the $n_{\mathrm{R}}$ resistive components is

$$
u_{R q}(t)=\sum_{c=1}^{n_{\mathrm{R}}}\left(\mathbf{D}_{R l}\right)_{q c} \cdot u_{R c}\left(i_{R c}(t)\right),
$$

where $u_{R c}\left(i_{R c}(t)\right)$ is voltage-current characteristic of the $c$ th resistive component. The voltage in the $q$ th current loop due to the $n_{\mathrm{L}}$ inductive components is

$$
u_{L q}(t)=\sum_{c=1}^{n_{\mathrm{L}}}\left(\mathbf{D}_{L l}\right)_{q c} \cdot \frac{\mathrm{d} \phi_{L c}\left(i_{L c}(t)\right)}{\mathrm{d} t},
$$

where $\phi_{L c}\left(i_{L c}(t)\right)$ is the flux-current characteristic of the $c$ th inductive component [11].

\section{HARMONIC-BALANCE APPROACH}

The HB approach allows obtaining the periodic steady-state solution by solving a unique, but larger and denser, system of algebraic equations. The $n$ coefficients in $\mathbf{X}(t)$ can be expressed as a truncated Fourier series considering a dc term and $n_{\mathrm{f}}$ frequencies $f_{k}, k=1,2, \ldots$, integer multiples of the fundamental frequency $f_{1}$ (period $T=1 / f_{1}$ ), or pulsations $\omega_{k}=2 \pi f_{k}$, for a total of $n_{\mathrm{h}}=2 n_{\mathrm{f}}+1$ harmonic basis functions (BFs), $h_{0}=1, h_{k}$, and $n \cdot n_{\mathrm{h}}$ unknown coefficients collected in column vectors $\mathcal{H}(t)$ and $\mathbf{X}^{\mathrm{H}}$ respectively [11]:

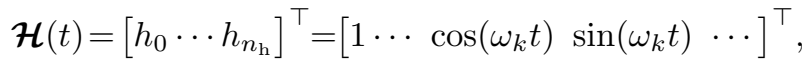

$$
\begin{aligned}
& \mathbf{X}(t)=\left(\mathbb{1} \otimes \mathcal{H}(t)^{\top}\right) \mathbf{X}^{\mathrm{H}},
\end{aligned}
$$

where $\otimes$ denotes the Kronecker product.

These $n_{\mathrm{h}}$ cosine and sine BFs are mutually orthogonal and coupled via their time derivative:

$$
\begin{gathered}
\frac{2}{T} \int_{0}^{T} \mathcal{H}(t) \mathcal{H}^{\top}(t) \mathrm{d} t=\mathbb{1} \\
\mathcal{Q}=\frac{2}{T} \int_{0}^{T} \mathcal{H}(t) \frac{\mathrm{d} \mathcal{H}^{\top}}{\mathrm{d} t} \mathrm{~d} t=\left[\begin{array}{cccc}
\cdots & \cdots & \cdots & \cdots \\
\cdots & 0 & \omega_{k} & \cdots \\
\cdots & -\omega_{k} & 0 & \ldots \\
\cdots & \ldots & \ldots & \ldots
\end{array}\right] .
\end{gathered}
$$

The ODEs (5) are weakly imposed using the $h_{j}(t)$ BFs [11]:

$$
\frac{2}{T} \int_{0}^{T}\left(\mathbf{M X}+\mathbf{N} \frac{\mathrm{d} \mathbf{X}}{\mathrm{d} t}\right) h_{j} \mathrm{~d} t=\frac{2}{T} \int_{0}^{T} \mathbf{F} h_{j} \mathrm{~d} t,
$$

leading to one system of $n \cdot n_{\mathrm{h}}$ algebraic equations, that in the linear case reads:

$$
\begin{aligned}
& \mathbf{M}^{\mathrm{H}} \mathbf{X}^{\mathrm{H}}=\mathbf{F}^{\mathrm{H}}, \quad \mathbf{M}^{\mathrm{H}}=\mathbb{1} \otimes \mathbf{M}+\mathcal{Q} \otimes \mathbf{N}, \\
& \mathbf{F}^{\mathrm{H}}=\frac{2}{T} \int_{0}^{T} \boldsymbol{H}(t) \otimes \mathbf{F}(t) \mathrm{d} t .
\end{aligned}
$$

\section{A. Treatment of Nonlinearity}

In case of nonlinearity, we can't use the Kronecker product for compacting the equations. The nonlinear HB system (14) is linearized by means of the Newton-Raphson (NR) method. At the material level, the harmonic differential reluctivity tensor depends on the differential reluctivity tensor which in turn depends on the harmonic component of the induction [11].

Analogously, the current-dependent lumped resistances (e.g. diodes) are characterized by harmonic differential resistances

$$
\frac{\partial u_{R q}^{k}}{\partial i_{l}^{j}}=\sum_{c=1}^{n_{\mathrm{R}}}\left(\mathbf{D}_{R l}\right)_{q c}\left(\mathbf{D}_{R l}\right)_{l c} \frac{\partial u_{R c}^{k}}{\partial i_{R c}^{j}}
$$

that depend on the differential resistances:

$$
\frac{\partial u_{R c}^{k}}{\partial i_{R c}^{j}}=\frac{1}{T} \int_{0}^{T} \frac{\mathrm{d} u_{R c}^{k}}{\mathrm{~d} i_{R c}^{j}} h_{k}(t) h_{j}(t) \mathrm{d} t .
$$

The current-dependent lumped inductances are characterized by harmonic differential fluxes

$$
\frac{\partial \phi_{L q}^{k}}{\partial i_{l}^{j}}=\sum_{c=1}^{n_{\mathrm{L}}}\left(\mathbf{D}_{L l}\right)_{q c}\left(\mathbf{D}_{L l}\right)_{l c} \mathcal{X}_{L c}^{k j}
$$

that depend on the differential reactances $\mathcal{X}_{L c}^{k j}$ :

$$
\mathcal{X}_{L c}^{k j}=\frac{1}{T} \int_{0}^{T} \frac{\mathrm{d} \phi_{L c}^{k}}{\mathrm{~d} i_{L c}^{j}} \frac{\mathrm{d} h_{k}(t)}{\mathrm{d} t} h_{j}(t) \mathrm{d} t .
$$

The time integration in (14) is performed numerically considering a sufficiently large number of equidistant and equalweighted time instants in $[0, T]$.

\section{Homogenization of Windings}

In (5) eddy currents are explicitly accounted for via a conductivity-dependent eddy-current matrix (classical FE) or via a frequency-dependent complex reluctivity [4] in the homogenized winding window (one stranded inductor) and a complex impedance replacing the dc resistance.

In the multi-harmonic case, we adopt a different proximityeffect complex reluctivity $\boldsymbol{\nu}_{\text {prox }}\left(f_{k}\right)$ and skin-effect impedance $Z_{\text {skin }}\left(f_{k}\right)$ per considered frequency in the HB approach [6]. The cosine and sine HB-BFs are coupled due to these effects. The matrix $\mathbf{M}^{\mathrm{H}}$ is modified by including $\boldsymbol{\nu}_{\text {prox }}\left(f_{k}\right)$ in the reluctivity-dependent stiffness matrix $\mathbf{S}(\nu)$ as:

$$
\left[\begin{array}{cccc}
\cdots & \cdots & \cdots & \cdots \\
\cdots & \mathbf{S}\left(\Re\left(\boldsymbol{\nu}_{\text {prox }}\left(f_{k}\right)\right)\right) & \mathbf{S}\left(\Im\left(\boldsymbol{\nu}_{\text {prox }}\left(f_{k}\right)\right)\right) & \cdots \\
\cdots & -\mathbf{S}\left(\Im\left(\boldsymbol{\nu}_{\text {prox }}\left(f_{k}\right)\right)\right) & \mathbf{S}\left(\Re\left(\boldsymbol{\nu}_{\text {prox }}\left(f_{k}\right)\right)\right) & \cdots \\
\cdots & \cdots & \cdots & \cdots
\end{array}\right],
$$

and by integrating $\boldsymbol{Z}_{\text {skin }}\left(f_{k}\right)$ in the circuit coupling blocks linking currents and voltages in stranded conductors:

$$
\left[\begin{array}{cccc}
\cdots & \cdots & \cdots & \cdots \\
\cdots & \Re\left(\boldsymbol{Z}_{\text {skin }}\left(f_{k}\right)\right) & \Im\left(\boldsymbol{Z}_{\text {skin }}\left(f_{k}\right)\right) & \cdots \\
\cdots & -\Im\left(\boldsymbol{Z}_{\text {skin }}\left(f_{k}\right)\right) & \Re\left(\boldsymbol{Z}_{\text {skin }}\left(f_{k}\right)\right) & \cdots \\
\cdots & \cdots & \cdots & \cdots
\end{array}\right]
$$




\section{Application ExAmple}

By way of validation, we consider a single-phase four-diode rectifier in Fig. 1 with a sinusoidal voltage supply $(50 \mathrm{~V}$ peak, $f=10 \mathrm{kHz})$, a filter inductor $L$, a filter capacitor $(C=10 \mu \mathrm{F})$ and a load resistance $R=100 \Omega$. The diodes are modelled by a piecewise linear characteristic, namely $R_{\text {on }}=10^{-1} \Omega$ in conducting mode $\left(i_{R c} \geq 0\right)$ and $R_{\text {off }}=10^{5} \Omega$ in nonconducting mode $\left(i_{R c}<0\right)$.

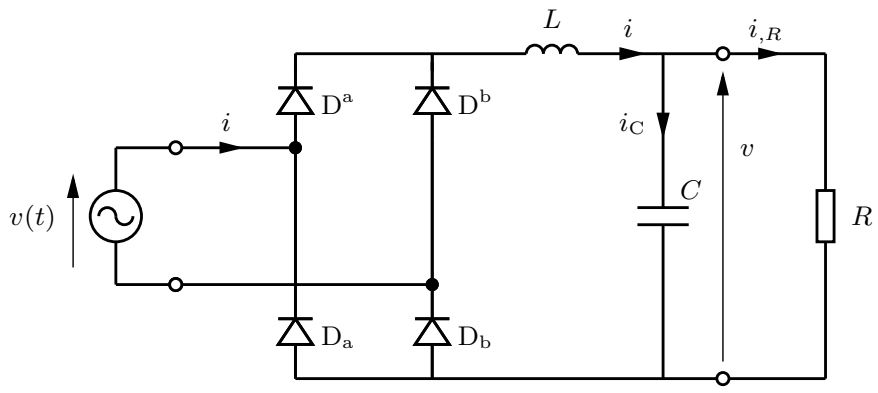

Fig. 1. Single-phase four-diode rectifier with a filter inductor modelled by finite elements with and without homogenization.

The inductor is a 120-turn coil with round copper conductors $\left(\sigma=60 \mathrm{MS} / \mathrm{m}\right.$, section $\left.1 \mathrm{~mm}^{2}\right)$ and square packing $(\lambda=0.65$, see Fig. 2). The total dc resistance and inductance are $R_{\mathrm{dc}}=0.19 \Omega, L_{\mathrm{dc}}=2.7 \mathrm{mH}$. The magnetic core ( $\mu_{r}=1000$, non-conducting $\sigma=0$ ) has a central $3 \mathrm{~mm}$ airgap. It is accurately modelled by FEs accounting for eddy-current effects (brute-force and homogenized approach, see Fig. 2).
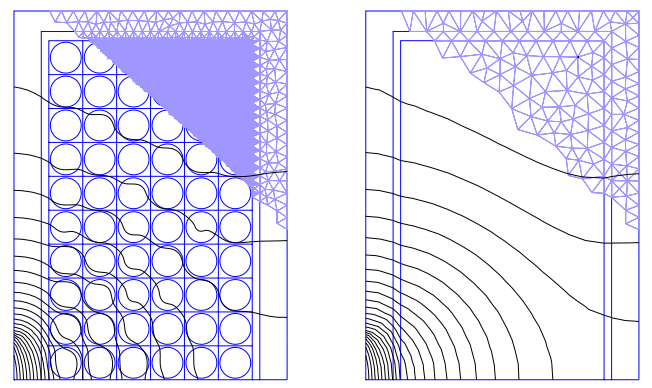

Fig. 2. Flux lines in winding domain obtained with the fine (left) and homogenized model (right) at $f=50 \mathrm{kHz}$. Axisymmetric model, half inductor. Detail of the meshes.

Time-stepping simulations (no homogenization) are carried out for validating the $\mathrm{HB}$ approach (with homogenization). The long transient is time-stepped with $\Delta t=T / 1000(250$ periods). HB calculations are carried out considering first only the dc term and the fundamental frequency (denoted by HB 2), and then gradually expanding the spectrum with harmonics, up to the 99th harmonic (HB 100).

At the ac-side of the rectifier, the current waveform at the voltage supply comprises only odd harmonics. The dc term and all harmonics are present in the diode currents, e.g. $D^{a}$. The corresponding current waveforms computed with the homogenized winding HB approach are compared to the reference TD result in Fig. 3. The sharp switching of diodes gives rise to high harmonics in the solution. Even though the convergence to the TD reference is evident, the discrepancy between the curves is still apparent for HB 100. The current waveforms at the dc-side are depicted in Fig. 4, the spectrum of these currents consists of the dc term and even harmonics. One observes that from HB 50 on, the agreement with the time-stepping results is excellent at both the inductor and load level.
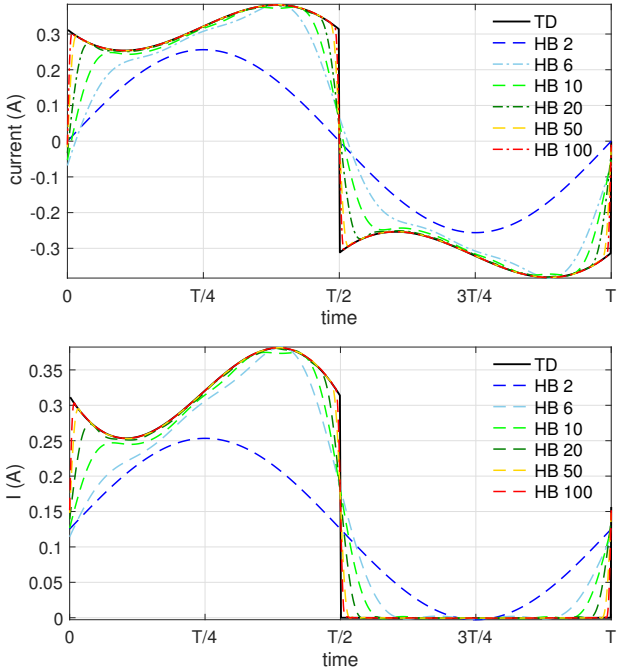

Fig. 3. Current waveforms at the ac-side: in the supply $i_{\text {ac }}$ (up) and the diode $D^{a}$ (down)
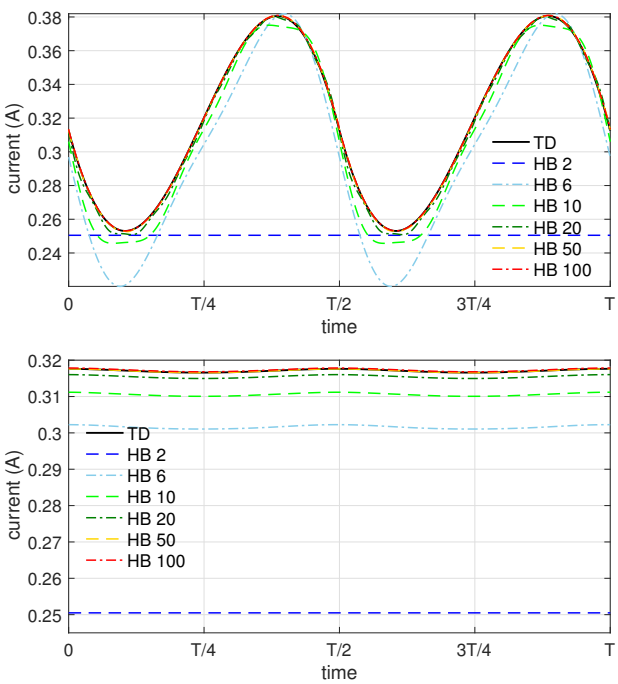

Fig. 4. Current waveforms at the dc-side: in the inductor $i_{\mathrm{d} c}$ (up) and the load $i_{\mathrm{dc}, R}$ (down).

The error made with the HB $n$ approach is measured in the time and frequency domain and represented in Figs. 5 and 6. The relative $L_{2}$-error in time in Fig. 5 is much smaller at the dc-side for both the inductor and load (under $1 \%$ with $n>20$ ), as the frequency content of the currents is much lower. The normalized error of the frequency components of the currents at the dc-side obtained with HB 2 to HB 100 is depicted in Fig. 6 as a function of the harmonic number. The error of the dc-component at the load is $20 \%, 4.8 \%, 2 \%, 0.5 \%$, 
$0.072 \%$ and $0.069 \%$ with HB 2, HB 6, HB 10, HB 20, HB 50 and HB 100, respectively. The other frequency components are accurately calculated as well. Similar behaviour is observed at the inductor level.

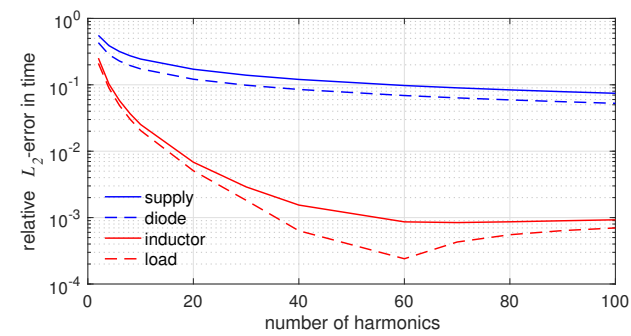

Fig. 5. Relative $L_{2}$-error, $\varepsilon_{n}=\frac{\left\|i_{\mathrm{TD}}-i_{\mathrm{HB}}\right\|_{2}}{\left\|i_{\mathrm{TD}}\right\|_{2}}$, vs. the number of harmonics $n$ of the harmonic-to-time current obtained with $\mathrm{HB} n, i_{\mathrm{HB}_{n}}(t)$, with regard to the reference steady-state TD solution, $i_{\mathrm{TD}}$, (one period).
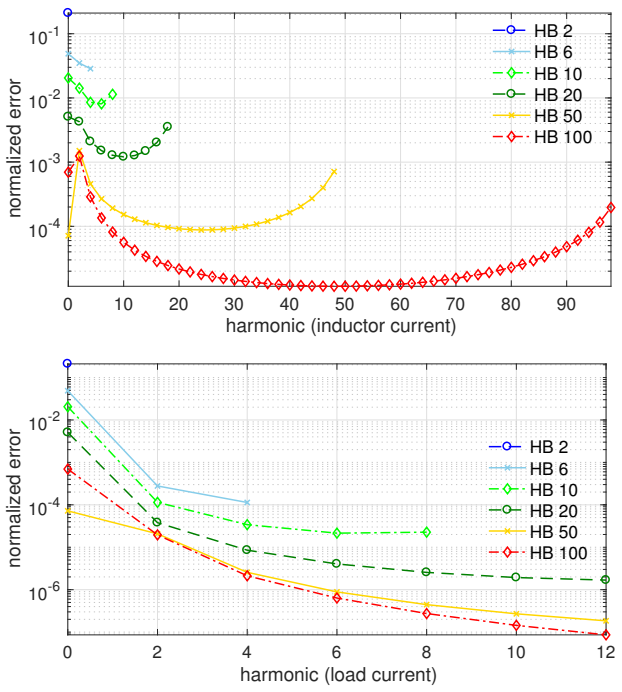

Fig. 6. Normalized error $\varepsilon_{n}\left(f_{k}\right)$ of the $f_{k}$ component of the current obtained with $\mathrm{HB} n, \boldsymbol{I}_{\mathrm{HB} n}^{k}$, with $\varepsilon_{n}\left(f_{k}\right)=\frac{\left|\boldsymbol{I}_{\mathrm{TD}}^{k}-\boldsymbol{I}_{\mathrm{HB} n}^{k}\right|}{\max \left(\left|\boldsymbol{I}_{\mathrm{TD}}\right|\right)}$, where $\boldsymbol{I}_{\mathrm{TD}}^{k}$ denotes the $k$ th frequency component of the complex representation $\boldsymbol{I}_{\mathrm{TD}}$ of the TD reference current $i_{\mathrm{TD}}(t)$. Up: at the inductor. Down: at the load.

Note the fill-in of the Jacobian matrix increases with the number of considered frequencies. However, the NR process with adaptive relaxation converges well, even in the presence of the diodes in the electrical circuit. If the NR process is initialized with the previous HB solution (with less frequencies), the number of NR iterations is considerably reduced and thus the computation time. In average, the NR iterations go from 50 to 4 when using initialization (prescribed tolerance $10^{-8}$ ).

The overall minimum computation time (on a $2.7 \mathrm{GHz}$ Intel Core i7 MacBook Pro with 16GB RAM) is roughly $110000 \mathrm{~s}$ $(30.5 \mathrm{~h})$ for the TD simulation ( 250 periods $\times 1000$ time steps per period $\times 3 \mathrm{NR}$ iterations per time step in average). For the HB simulations, solution (with adaptive relaxation, NR iterations indicated between parenthesis) is obtained in $0.96 \mathrm{~s}$ (10), $2.5 \mathrm{~s}$ (11), $3.5 \mathrm{~s}$ (7), $4 \mathrm{~s}(5), 12.8 \mathrm{~s}$ (4), and $41.7 \mathrm{~s} \mathrm{(3)} \mathrm{for}$ HB 2 to HB 100, respectively (HB 2 is not initialized, HB 6 to HB 100 are started from HB 5 to HB 99, respectively).

\section{Conclusions}

We have tackled a nonlinear circuit-field problem with a multi-harmonic approach. The filter inductor in the circuit is modeled by means of a HB-FE homogenization approach accurately accounting for the eddy-current effects. Four dimensionless frequency-dependent coefficients, characterizing the winding, are directly introduced in the HB-FE model via a multi-harmonic reluctivity and impedance.

HB results with homogenized winding are validated with classical time-stepping without homogenization. The proposed approach allows for high accuracy and reduced computational cost, what enables a strong circuit-field coupling. Though the considered inductor is axisymmetric, the applicability of the method is general.

The nonlinear coupled system of equations is successfully solved by means of the NR method. The HB results converge well to the (more accurate) TD results. The number of NR iterations can be significantly reduced by adaptive relaxation and initialization. Research on more suitable solvers for the HB approach is on-going.

\section{ACKNOWLEDGMENT}

Work supported by the Belgian Science Policy (IAP P7/02) and the Walloon Region under WBGreen-FEDO grant (convention n. 1217703).

\section{REFERENCES}

[1] D. C. Meeker, "An improved continuum skin and proximity effect model for hexagonally packed wires," J. Comput. Appl. Math., vol. 236, no. 18, pp. 4635-4644, 2012.

[2] G. Meunier, V. Charmoille, C. Guérin, P. Labie, Y. Maréchal, "Homogenization for Periodical Electromagnetic Structure: Which Formulation?," IEEE Trans. Magn., vol. 46, no. 8, pp. 3409-3412, 2010.

[3] H. Igarashi, "Semi-Analytical Approach for Finite-Element Analysis of Multi-Turn Coil Considering Skin and Proximity Effects," IEEE Trans. Magn., vol. 53, no. 1, pp. 1-4, article \#7400107, 2017.

[4] J. Gyselinck, R. V. Sabariego and P. Dular, "Time-domain homogenization of windings in 3-D finite element models," IEEE Trans. Magn., vol. 44, no. 6, pp. 1302-1305, 2008.

[5] K. Niyomsatian, J. Van den Keybus, R. V. Sabariego and J. Gyselinck, "Frequency-domain homogenization for litz-wire bundles in finite element calculations," In Proceedings of the 18th European Conference on Power Electronics and Applications (EPE'16 ECCE Europe), Karlsruhe, Germany, 2016, pp. 1-10.

[6] R. V. Sabariego, J. Gyselinck, "Eddy-Current-Effect Homogenization of Windings in Harmonic-Balance Finite Element Models," IEEE Trans. Magn., vol. 53, no. 6, pp.1-4, article \#7206304, 2017.

[7] S. Yamada and K. Bessho, "Harmonic field calculation by the combination of finite element analysis and harmonic balance method," IEEE Trans. Magn., vol. 24, no. 6, pp. 2588-2590, 1988.

[8] H. De Gersem, S. Vandewalle, and K. Hameyer, "Krylov subspace methods for harmonic balanced finite element methods," In Lecture Notes in Computational Science and Engineering, pp. 387-396, 2001.

[9] F. Bachinger, U. Langer, and J. Schöberl, "Numerical analysis of nonlinear multiharmonic eddy current problems," Numer. Math., vol. 100, no. 4, pp. 593-616, 2005.

[10] S. Ausserhofer, O. Biro, and K. Preis, "An efficient harmonic balance method for nonlinear eddy-current problems," IEEE Trans. Magn., vol. 43, no. 4, pp. 1229-1232, 2007.

[11] J. Gyselinck, P. Dular, C. Geuzaine, W. Legros, "2D harmonic balance FE modelling of electromagnetic devices coupled to nonlinear circuits," COMPEL, vol. 23, no. 3, pp. 800-812, 2004.

[12] H. De Gersem, K. Hameyer, T. Weiland, "Field-circuit coupled models in electromagnetic simulation," J. Comput. Appl. Math., vol. 168, no. 1-2, pp. 125-133, 2004.

[13] A. Bartel, S. Baumanns, S. Schöps, "Structural analysis of electrical circuits including magnetoquasistatic devices," Applied Numerical Mathematics, vol. 61, pp. 1257-1270, 2011. 